\title{
LA ARGUMENTACIÓN A LA LUZ DE LA FILOSOFÍA DE LA BIOLOGÍA
}

\author{
Cristián Santibáñez Yáñez \\ Centro de Estudios de la Argumentación y el Razonamiento \\ Facultad de Psicología, Universidad Diego Portales \\ critian.santibanez@udp.cl
}

\begin{abstract}
Resumen / Abstract
Se ofrece una respuesta inicial a la pregunta sobre el recorrido evolutivo de la competencia argumentativa. Se asume decididamente la hipótesis de la intencionalidad colectiva y la cooperación como rasgos estructurales que permiten entender la argumentación como un fenómeno normativo. Se concluye que la argumentación fue producto de una presión selectiva para la multiplicación de representaciones alternativas provenientes de una mayor cantidad de agentes de un mismo o diferente grupo. La presión evolutiva seleccionó la comunicación de buenas razones a través del conflicto verbal y permitió, al agente individual, generar reputación, lo que va en su directo beneficio reproductivo.

Palabras clave: cooperación, evolución, intencionalidad colectiva, mutualismo, normatividad.
\end{abstract}

\section{ARGUMENTATION IN THE LIGHT OF PHILOSOPHY OF BIOLOGY}

An initial answer to the question of the evolutionary path of the argumentative competence is offered. The hypothesis of collective intentionality and cooperation as structural features that allow to understand argumentation as a normative phenomenon is assumed. The paper concludes that argumentation was a product of selective pressure for the multiplication of alternative representations offered by a larger number of agents of the same or a different group. The evolutionary pressure selected the communication of good reasons by means of verbal conflict, and allowed the individual agent to generate a reputation that, in turn, profits her direct reproductive benefit.

KEY WORDS: cooperation, evolution, collective intentionality, mutualism, normativity.

\section{Introducción}

R⿴囗十 La argumentación ha recibido explicación detallada desde hace tiempo (incluso mucho antes de que se le bautizara como campo teórico propio), y desde varios subcampos y/o corrientes filosóficas, tales como el retórico, el lógico, el dialéctico, el pragmático, el analítico, el epistémico, el mental o cognitivo, el semántico, por nombrar algunos que ya intuitivamente se le reconocen. Ciertamente, y dependiendo de la literatura específica y autor/a consultados, estos subcampos se mezclan para efectos de construir una teoría con alto rendimiento explicativo. Se puede ver al respecto el acercamiento 
epistémico-pragmático de Toulmin (1958)1, la visión retórica de Perelman (1958), el ángulo pragma-dialéctico de van Eemeren y Grootendorst (2004), el acento de la lógica de diálogos de Barth y Krabbe (1982), el desarrollo semantista de Naess (1953). La lista puede continuar así como se detalle el énfasis, como por ejemplo se observa en Rigotti (2009) para el caso de una combinación entre retórica y pragma-dialéctica, en Kock (2007) para efectos de un ángulo híbrido entre retórica y dialéctica, en Walton (2013) cuando se trata de neo-dialéctica, en Tindale (2004) para aprender sobre retórica y algo de cognición, en Johnson (2000) si se trata de evaluar la combinación entre dialéctica y lógica, en Freeman (2005) a propósito de epistemología y lógica informal, etc.

Hasta donde se tienen noticias, la filosofía de la biología, un subcampo filosófico relativamente novel, no ha sido incluido en estos esfuerzos teóricos, a pesar de que ha producido análisis renovado de una variedad amplia de fenómenos elementales en la filosofía, tales como los problemas morales de engaño y autoengaño, o los problemas de la filosofía de la ciencia relativos a los cambios científicos, o los problemas relacionados con categorías como función, estructura, confianza, intencionalidad, representación, entre muchos otros ${ }^{2}$.

En virtud de esta despreocupación, en lo que sigue se ofrecerán distinciones sobre algunas dimensiones de lo argumentativo a la luz de las discusiones que se han realizado en el seno de la literatura de la filosofía de la biología y otras reflexiones disciplinarias afines. En particular, se abordará en la sección 2 la dimensión normativa sine qua non de la argumentación analizando el concepto de intencionalidad colectiva en combinación con la propuesta cognitivo-culturalista; en la sección siguiente, especial atención se dará al problema de la cooperación en tanto infraestructura elemental a partir de la que el encuadre culturalista toma cuerpo, dividiendo la crítica en las categorías que la filosofía de la biología ha propuesto como sub-tipos de comportamientos cooperativos; en la sección 4, que corresponde a las conclusiones, el trabajo se centrará en explicar la argumentación como proceso objetivador, tanto como producto de lo discutido en las secciones anteriores como de apreciar el acto de dar razones como una actividad consecuencia de la acumulación cultural y tecnológica, tal como la filosofía de la biología tiende a sugerir.

1 Retrospectivamente se puede tomar la visión de Toulmin como parte del campo de la teoría de la argumentación, pues él mismo señaló (Toulmin 2004) que su intento fue parte de una discusión en epistemología y filosofía de la ciencia de su tiempo.

2 El trabajo de Mercier y Sperber (2011), considerado un nuevo impulso cognitivo y evolutivo para explicar el razonamiento, primero no incursiona decididamente en la literatura especializada en filosofía de la biología, y segundo, no trata específicamente la argumentación, sino que hace un análisis argumentativo del razonamiento. Para una crítica de la postura de Mercier y Sperber, véase Santibáñez (2012). 


\section{Normatividad argumentativa: intencionalidad y recursión}

La argumentación es por definición una actividad normativa: cuando discuto con alguien considero que tengo un punto de vista más correcto, sólido o verdadero y más certeramente justificado que el de mi oponente, pues si pensara lo opuesto cometería flagrantemente una contradicción pragmática; y desde la perspectiva de poner en duda el punto de vista de otro, espero que aquel ofrezca razones de peso o, al menos, que ellas justifiquen suficientemente lo que mantiene. Como se observa, argumentar es materia de logro, de estándares, de criterios, de evaluación. La teoría de la argumentación ha reflexionado bastante sobre tales criterios, pero ¿cómo llegamos a ser una especie que su mayor logro comunicativo es tener la expectativa constante de transar creencias justificadas?

Obsérvese que responder la pregunta es ya afirmar su contenido: lograr una buena respuesta para satisfacer la expectativa. Y parece ser que, como lo he sostenido en otras ocasiones (Santibáñez 2015), la mejor respuesta que se puede dar proviene de la unión entre la comprensión de intenciones y la explicación del funcionamiento de la cognición cultural. En esto confluye un amplio abanico de filósofos, lingüistas y psicólogos (Bermúdez 2003; Boehm 2012; Bratman 2014; Carruthers 2006, 2011; Corbalis 2011; Searle 2010; Sterelny 2012; Tomasello 2014; Tomasello et al. 2005).

Es un buen comienzo apreciar la explicación que recientemente Tomasello (2014, pp. 110-111) ha propuesto, desde un punto de vista evolutivo, respecto de por qué los agentes humanos damos razones:

With modern humans and their skills of conventional linguistic communication, we get to full-blooded reasoning, where "reasoning" means not just to think about something but to explicate in conventional form -for others or oneself-the reasons why one is thinking what one is thinking... The key point is that arguing in this way assumes a cooperative context. As Darwall (2006, p. 14) puts it: "It is only in certain contexts, say, when you and I are trying to work out what to believe together, that either of us has any standing to demand that one another reason logically.

Ciertamente, Tomasello no es el primero en tratar de explicar el razonamiento desde una perspectiva evolutiva. Desde la famosa prueba de selección de Wason, muchos esfuerzos renovados se han hecho por un variado espectro de autores. Lo que la mayoría de estos análisis tienen en común es que ofrecen descripciones de los tipos de mecanismos que supuestamente subyacen al razonamiento y la toma de decisiones, en vez de ver la práctica de argumentar como un comportamiento social distintivo.

Y lo distintivo de la argumentación es que es un comportamiento en relación, uno en el que un punto de vista avanzado por un agente es rechazado, dudado o desafiado por otro agente (o muchos otros), y que entonces ciertas razones son ofrecidas para apoyar tal punto de vista, razones que son nuevamente desafiadas..., y así hasta que el desacuerdo se resuelva (Toulmin, Rieke \& Janik 1979; van Eemeren \& Grootendorst 2004; Tindale 2004; Walton 2013). Si tomamos la explicación de Tomasello de dar 
razones, esta definición básica es parcialmente capturada y, lo que es más importante, no refiere decididamente al carácter enteramente social de la competencia de argumentar. Lo que se extraña en la existente literatura de la psicología del razonamiento, los acercamientos cognitivos y la teoría de la toma de decisiones sobre el razonamiento, es una explicación genuina y enteramente colectivista de la actividad de intercambiar razones y no solamente un análisis psicologista de un acto o envío solitario (one-shoot) de razones como justificación retrospectiva de una decisión, creencia o acción; además, esta literatura observa la actividad de dar razones como una habilidad mental al servicio de la adaptación individual, lo que es una explicación bastante desbalanceada de un comportamiento en el que al menos dos partes juegan roles decisivos.

El punto de partida es la evidencia incuestionable de que los seres humanos participamos en actividades colaborativas que suponen metas compartidas y planes de acciones socialmente coordinados. Las interacciones de este tipo requieren no solo entender las metas, intenciones y percepciones de otros, sino que también requieren de una motivación por compartir estos estados con otros por medio de representaciones cognitivas dialógicas. La intencionalidad compartida es un logro evolutivo que hoy lo damos por descontado, pero supuso pasar de la comprensión de la intención del otro al reconocimiento inferencial recursivo, vale decir, de saber que tú sabes que es tu intención, y que ciertas actividades -como el lenguaje-son solo posible porque se deben a un equilibrio colaborativo en el que los participantes tienen una meta o compromiso compartido. Cuando se mantienen en el tiempo estas prácticas se obtiene lo que Searle (2010) denomina hechos institucionales, cuya fórmula lógica es X cuenta como Y en el contexto $C$, en la que concurren los agentes vía roles que se van intercambiando así como ocurran los reconocimientos y usos.

Antes que fuera posible representarse lógicamente los hechos institucionales, cierta madurez cognitiva tuvo que alcanzarse. Si se sigue una posición filogenética y culturalista (Tomasello 1999, 2008, 2014), la definición de cognición humana queda más acotada. En esta perspectiva, la cognición humana es producto de un proceso evolutivo social en el que la manipulación de información a través de representaciones mentales es consecuencia del intercambio entre miembros que comparten escenarios atencionales conjuntos. Cuando los agentes comparten escenarios atencionales y saben que el recipiente de una comunicación está evaluando su comportamiento comunicativo, para efectos de comprensión por ejemplo (también puede ser para efectos de defensa de grupo), el comunicador debe acoplarse con el conjunto de creencias, representaciones o perspectivas que el recipiente tiene. De modo que la destreza cognitiva de base cultural es como sigue: el agente incluye en su perspectiva la del otro, automonitoreándose y autorregulándose (las más de las veces de forma automática e intuitiva) ${ }^{3}$. Esta competencia mental es por defecto dialógica. Es lo que Tomasello (2014, p. 79) llama cognición cooperatizada, que permite institucionalización (Searle 1995) y convencionalización (Lewis 1969).

3 Véase respecto del sistema dual de la mente, en el que el razonamiento intuitivo se describe en detalle, Evans (2010), Kahneman (2011). 
¿En qué sentido lo normativo cabe en esta forma de concebir las cosas? En que el agente satisface las expectativas mutuas de los que integran un grupo. ¿Qué expectativas mutuas? Mantener prácticas institucionales beneficiosas para la comunidad, asegurar los mecanismos que han otorgado fluidez material y emocional, etc. La única forma de lograrlo es a través de extrapolar los criterios que juzgan comportamientos, pasar de la relación de segunda persona (tú y yo), a relaciones juzgadas por criterios transpersonales, de relaciones genéricas, depositarias de los estándares objetivos que evalúan acciones.

¿De qué manera la facultad de argumentar se inscribe en esta competencia general cooperativa y de intencionalidad compartida? Una respuesta posible es que la argumentación tiene como función propia propiciar la transacción de creencias, $u$ otros estados intencionales, que son mutuamente beneficiosos para todos los miembros de un grupo y que, desde el punto de vista comunicativo, son capaces de orientar la toma de cursos de acción adecuados. El motor para que la facultad se manifieste es el conflicto, el cual se controla precisamente porque se canaliza a través de una coordinación pacífica de las representaciones controversiales en el seno de un mismo grupo. El mecanismo argumentativo que se incorporó como destreza cognitiva dio la posibilidad a los menos talentosos o poderosos físicamente de un grupo, de cambiar la dirección de las creencias y acciones.

Parafraseando la fórmula recursiva inferencial de la comprensión de intenciones, la competencia argumentativa parte del diseño en que los agentes saben que el otro sabe que tú sabes que él evalúa tus razones, por lo que la comunicación de buenas razones es una presión selectiva. Como bien señala Skyrms (2004, 2014), era improbable que comportamientos engañosos constantes entre agentes se hubiesen mantenido, porque a la larga dañan a todos los miembros imposibilitando alcanzar la formación de grupos en los que la conducta recíproca altruista fuera dominante y equilibrada (Bowles \& Gintis 2011). La idea de equilibrio se utiliza aquí como un estado relacional entre agentes que saben que tienen cierta paridad cognitiva. Una vez que tal equilibrio se introduce, la argumentación deviene en una estrategia evolutivamente estable. El agente que es capaz de comunicar representaciones, creencias y perspectivas que resuelven problemas o mantienen beneficios obtendrá una gran reputación, y aquellos que tienen este reconocimiento podrán administrar la producción, distribución y sanción de razones y pretensiones, vale decir, ejercerán poder.

En la teoría de la argumentación contemporánea más estándar, un tímido acercamiento a una visión culturalista, pero lamentablemente en absoluto filogenética, es la de Gilbert (2007), para quien justamente la dimensión normativa sine qua non de la argumentación emerge de su dinámica colectiva, en la que las metas que los agentes tienen se ven controladas por las metas de los otros con quienes nos comunicamos. Esta relación controla dialécticamente a sus participantes y, más importante aún, controla el equilibrio entre metas menos explícitas que uno puede tener cuando avanza un argumento y el campo de alternativas de movimientos argumentativos. El contexto, añade Gilbert, es también una fuente natural de normatividad, ya que determina qué tipo de información es incluida o excluida para analizar y evaluar un argumento. Asimismo, otra fuente de normatividad emana del ethos del hablante, esto quiere decir, de las preconcepciones 
vinculadas al comportamiento moral conocido del hablante, que deviene en niveles de confianza y credibilidad, y que más arriba habíamos apuntado como reputación. Como se venía discutiendo, la normatividad natural proviene de la presión social de coordinarnos con otros ejerciendo una competencia que se mantiene por la exposición más o menos reflexiva de dar-buenas-razones.

Es necesario enfatizar que la argumentación ha sido siempre parte de un esfuerzo cooperativo para resolver diferencias de opinión, puesto que si las controversias hubiesen sido resueltas solo a través de actos violentos, se hubiesen reducido las posibilidades de supervivencia o el crecimiento de los grupos. Al mismo tiempo, resolver conflictos solo con el uso de órdenes expresadas por los líderes de un grupo (y con la garantía implícita del uso de la fuerza en casos de desobediencia), hubiese reducido las probabilidades de optar por soluciones más óptimas que los menos talentosos, o menos poderosos o más jóvenes del grupo, pudieran proponer. De modo que cooperación, intencionalidad colectiva y un sistema comunicativo estable son los elementos básicos de esta facultad humana robusta ${ }^{4}$.

La argumentación es la única forma de producir cambios en las representaciones, pues es el comportamiento comunicativo que preserva el conflicto verbal o simbólico como fuerza central, vale decir, el mecanismo social que asegura el constante intercambio de diferentes estados de cosas, lo que se logra conflictuando continuamente nuestras representaciones ${ }^{5}$. Del mismo modo, sostener que la argumentación establece creencias $\mathrm{y}$ otros estados intencionales mutuamente beneficiosos, sigue de cerca un antecedente evolutivo básico, el que la etología ha identificado en la comunicación entre señales, esto es, una acción comunicativa que coevoluciona entre el que envía la señal y el que la recibe, acto en que ambos se benefician del intercambio de señales (Griebel \& Oller 2008; Maynard-Smith \& Harper 2003). Cada vez que hay un conflicto y los agentes envueltos en él sienten la necesidad de disolverlo, entonces la argumentación emerge ${ }^{6}$.

\section{Cooperación: bases de la normatividad argumentativa}

Recientemente, ha habido un gran esfuerzo por acentuar la idea de que la cooperación es un mecanismo sustancial del desarrollo evolutivo humano. El filósofo Sterelny (2012), por ejemplo, publicó sus Jean Nicod Lectures centrándose (como en otras de

4 Para un estudio empírico reciente sobre la robustez de la facultad argumentativa, véase Mercier, Deguchi, van der Henst \& Yama (2015).

5 Los biólogos evolucionistas recuerdan que una habilidad adaptada no siempre se adapta perfectamente al ambiente. La facultad argumentativa no debería ser vista como una excepción.

6 Esta definición es en algo similar a la perspectiva de Godfrey-Smith y Yegnashankaran (2011), en relación con el razonamiento, en el sentido de que es deliberativa en función y dialógica en estructura y origen. 
sus publicaciones, 2003), en la explosión cooperativa que tuvo lugar, de acuerdo con algunos estudios arqueológicos, hace 400 mil años, cuando los Neandertales ya eran capaces de cazar en grupos, con armas relativamente sofisticadas y con un estudio de los movimientos y hábitos de animales medianos y grandes. Este comportamiento cooperativo para Sterelny explicaría en gran parte el camino evolutivo de la complejidad cognitiva y social humana.

De modo que la cooepración puede ser vista como una selección natural para el éxito adaptativo. En esto último, por su generalidad, hay acuerdo. Sin embargo, a medida que avanza la investigación en biología evolutiva, psicología evolutiva y ciencias de la cognición, algunas posiciones observan más bien este fenómeno como un tipo específico de selección cultural (Henrich \& Henrich 2007; Richerson, Boyd $\&$ Henrich 2003) ${ }^{7}$, en una coevolución gen-cultura, mientras que otros, con un énfasis más psicológico, señalan que la cooperación como estado psicológico es expresión de un mutualismo colaborativo (Tomasello, Melis, Tennie, Wyman \& Herrmann 2012; Tomasello et al. 2009; Tomasello 2008).

Probablemente, en la historia del siglo XX de la reflexión sobre la materia (asumiendo caritativamente que antes del siglo XX hubo una reflexión sostenida sobre el tópico), habría que comenzar por dar crédito, una vez más, al trabajo conjunto de Axelrod y Hamilton (1981), y al volumen de Axelrod (1997), en los que se ofreció evidencia para sostener que la conducta cooperativa, al menos en la escena competitiva de juegos, tiende a la larga a crear una relación estable de cooperación, reduciendo la importancia y recurrencia de los movimientos iniciales de un agente en un juego tendiente al egoísmo y búsqueda de beneficio individual.

La literatura al respecto sigue acuñando y combinando distintos términos para distinguir este fenómeno; así por ejemplo, Bowles y Gintis (2004; Bowles \& Gintis 2011) han puesto un enorme énfasis en las nociones de reciprocidad fuerte y preferencias sociales en virtud de interacciones repetidas que moldean un tipo de psicología del agente humano que probablemente evolucionó a partir de la selección grupal. Por otra parte, un lugar también especial en la dicsuión contemporánea tiene la asociación entre castigo y conducta cooperativa (Richerson \& Boyd 2005), asociación que intenta explicar la interdependencia entre ambos fenómenos.

Nuevamente, partamos del banal hecho de que nosotros los mortales que vivimos en sociedad damos por sentado que cooperamos, pues evidencia de ello se encuentra en cada acto social, desde la espera ordenada que hacemos para pagar las compras del supermercado, cooperando así en el entendimiento tácito de seguir un orden en el tráfico comercial, hasta la confianza ciega que tenemos, precisamente, en que el otro

Se señala que el trabajo de Wynne-Edwards (1962) fue uno de los primeros esfuerzos explícitos en sentar la idea de una selección grupal y cultural. La propuesta de WynneEdwards fue atacada enérgicamente, y solo a partir de la década de 1980 esta idea recibió un nuevo impulso a partir del modelamiento matemático del comportamiento de grupos. 
hará lo mismo, incluso en aquellos contextos diseñados (cooperativamente) para dirimir desacuerdos, como es el caso de un tribunal.

Como ya se ha dicho, de acuerdo con una convergencia de autores (Dugatkin 2006; Godfrey-Smith 2009; Skyrms 2004; Sterelny 2012), Hamilton fue una piedra angular que logró reposicionar la discusión en torno al problema del altruismo y las unidades de selección. En particular, al insistir en la selección por parentesco (Kin selection), Hamilton trató de explicar la supervivencia de la estrategia altruista que, de forma sintética (Godfrey-Smith 2009: 115), se basa en la idea de que un individuo aumenta la representación de sus propios genes en generaciones futuras ayudando a otros en su reproducción, sobre el entendido de que aquellos otros portan genes similares al actor altruista (Dahanukar \& Watve 2009). Por su parte, la hipótesis de la selección de grupo sostiene que si bien a los individuos egoístas les va mejor que a los altruistas dentro de los grupos, en algunos casos el altruismo florece porque los grupos con muchos altruistas son más productivos en promedio que los grupos con egoístas en predominio ${ }^{8}$.

Esta forma de cooperación, como se observa, es específica y relativa a la reproducción, pero desde el punto de vista social y cultural, la cooperación representa una estrategia potente de adaptación porque permite una división del trabajo más inteligente y una coordinación en torno a la información (búsqueda, mantención y traspaso) más fina (Sterelny 2003, pp. 124ss). Obviamente, la cooperación es una adaptación difícil, la tentación al engaño egoísta es poderosa, tal como mostró el juego del dilema del prisionero en el que se analizó el comportamiento automático de agentes que buscan un beneficio a corto plazo. Pero no toda cooperación está sujeta al engaño automático de agentes egoístas, pues el intercambio lingüístico requiere una estabilidad que elimina el engaño egoísta. Por lo pronto, entonces, es necesaria una definición más exacta de cooperación y distinguir algunos tipos prototípicos de este comportamiento que, definitivamente, dieron posibilidad a la competencia y comportamiento argumentativos.

\subsection{Cooperación}

La cooperación ocurre cuando los individuos actúan en conjunto para benificio mutuo (Zimmerman, Mcelreath \& Richerson 2003: 155). Actuar en conjunto no significa aquí, por cierto, actuar en un mismo lugar en un momento preciso y específico. Se puede

8 Como ya se ha dicho, la discusión en torno a las unidades de selección y la hipótesis de selección de grupo es enorme en términos de literatura y posiciones. Tampoco un clásico como el de Dawkins (1976) se escapa, y de hecho su posición es una muy interesante porque provee de otra salida, que el propio autor denomina como alternativa, al poner el acento del debate en la selección por gen y no grupal o individual (Dawkins 2006, p. 7). Por otra parte, es llamativo notar que en la introducción a la edición 30 de su clásico (2006), Dawkins sostenga que el título, ahora retrospectivamente, pudo ser El gen cooperativo, en vez de El gen egoísta. 
colaborar a distancia, y en tiempos distintos. Pero del mismo modo, por supuesto, para determinados tipos de cooperación, estar juntos en un mismo momento hace una gran diferencia.

De acuerdo con West, Griffin y Gardner (2007), existe algo de confusión en toda la terminología asociada a la categoría cooperación, que tiene en biología evolutiva resultados negativos. Por ejemplo, estos autores, que desarrollan su mirada desde la biología evolutiva, dan una definición distinta a la de Zimmerman, Mcelreath y Richerson (quienes cultivan una mirada desde la psicología y la antropología evolutiva), señalando que cooperación es un comportamiento que provee un beneficio a otro individuo (el receptor), y seleccionado, precisamente, por sus efectos beneficiosos en el receptor (West, Griffin \& Gardner 2007, p. 416). En cualquier caso, y más allá de esta discrepancia incial existente entre estos ángulos de análisis, la pregunta que debe contestarse es: ¿por qué un agente debería desarrollar un comportamiento costoso en su realización sin beneficio aparente? La respuesta ha dividido a los teóricos en la materia: algunos sostienen que la razón es que produce un beneficio directo de adaptación, y otros que produce un beneficio indirecto de adaptación (Hamilton 1996). En la primera línea de explicación, el factor recompensa (Frank 1988) juega un rol importante, ya que tal recompensa puede signifcar la selección para el encuentro de pareja y asegurar reproducción; mientras que en la segunda línea de explicación, el argumento es que el beneficio es indirecto ya que el benificio directo es para quien luego carga el gen de la cooperación, el receptor. Precisamente este último argumento es el que ha propiciado mayor proliferación semántica, es decir, mayor cantidad de conceptos relacionados, los que evidenciarían una serie de subcomportamientos dentro de la categoría cooperación. Algunos de ellos se comentan a continuación.

\subsection{Mutualismo}

El mutualismo, en breve, es aquel comportamiento de cooperación, o ayuda, respecto del que los costos de producción son menores a los beneficios de la producción, o dicho de otra forma, es aquel comportamiento a partir del que los agentes derivan un beneficio de adaptación, es decir, beneficio mutuo (que tiene la fórmula: $+/+$ ). Para distinguirlo cabe comparar con el altruismo, que es aquel comportamiento cuyos costos de producción corren por cuenta del actor y cuyos beneficios son para el receptor (-/+). Pero el término mutualismo (Krebs \& Davies 1993; Ratnieks 2006) también carga con problemas semánticos. Puede ser el caso que se considere bajo mutualismo (dentro de una misma especie) la descripción del efecto de un solo comportamiento en el actor y el receptor, pero al mismo tiempo, mutualismo (para el caso de agentes de especies distintas) el efecto que cada individuo tiene sobre el otro.

En la evolución de la forma de la comunicación humana, el mutualismo, de acuerdo con Tomasello (2008), ha jugado un papel importante. Tomasello (2008; et al. 2009), después de muchos años de estudios experimentales con chimpancés e infantes acumulando gran cantidad de observaciones y datos, señala que la comunicación cooperativa entre humanos tiene, al menos, tres grandes motivadores que han 
evolucionado tanto desde el punto vista filogenético como ontogenético: requerir, informar y compartir. Ambos, comunicador y receptor, asumen mutuamente que el mensaje enviado tendrá beneficios individuales y colectivos. Como sostiene Tomasello (2008, p. 88), el comunicador conoce de tales beneficios y se asegura de que el receptor lo sepa, enfatizando que está al tanto del requerimiento del otro, enviando información relevante al otro, o compartiendo un hallazgo ventajoso para el otro. Son precisamente estos rasgos generales de la comunicación humana, que evolucionó bajo la presión selectiva de la cooperación, que la argumentación viene a asegurar.

Desde el punto de vista evolutivo en torno a los cambios psicológicos del linaje humano en este contexto de comportamiento comunicativo mutualista, lo anterior significa que desde la dimensión de la producción del mensaje (el comunicador), los humanos deben: 1) comunicarse con otros o de lo contrario se desarrollarían patologías, 2) requerir cosas razonables o no habrá respuestas positivas, 3) comunicar cosas pertinentes o serán catalogados como desadaptados socialmente; y desde la dimensión de la comprensión, se requiere de quien recibe que: 1) participe de la comunicación o pronto desarrollará también una patología, 2) debe ayudar, aceptar ayuda e información, de lo contrario arriesgará extrañamiento social; las dos dimensiones del proceso comunicativo es, y crea, simplemente, un mutualismo de expectativas (que puesto en la arena pública más llana se trata de derechos y obligaciones). La importancia, entonces, es que en estas bases mutualistas radica la normatividad de lo humano, incluida la argumentativa obviamente. Nótese que este normatividad tiene una base cognitiva, es decir, el desarrollo paulatino de capacidades motoras y cerebrales de compartir intención.

\subsection{Altruismo}

Fehr y Fischbacher (2003) parten de un hecho básico: en el mundo viviente la cooperación es más bien una excepción, y entre los que forman parte de esa excepción están los insectos sociales, primates y, afortunadamente, nosotros los humanos. La respuesta a este enigma para estos autores está en los patrones únicos del altruismo que exhibe el humano. Y el patrón fundamental está encapsulado en la reciprocidad fuerte. La reciprocidad fuerte es una combinación entre el altruismo que premia y el altruismo que castiga. De acuerdo con la teoría, los reciprocadores fuertes tienden a premiar al cooperador, y castigan sin miramientos al egoísta; sin embargo, parece ser que lo hacen (premiar y castigar) por un interés individual de largo plazo.

El altruismo tiene una fórmula -/+, es decir, costos para el altruista, ventajas para el beneficiario. Pero, ¿qué pasa cuando el actor altruista desarrolla un acto costoso que tiene beneficios para él en el largo palzo? Los especialistas (West, Griffin \& Gardner 2006, 2007; Hwanga \& Bowles 2012) en este caso hablan de un beneficio mutuo por lo que, consideran, no se trata de altruismo. ¿Qué pasa cuando el actor coopera altruísticamente porque en el pasado el beneficiario cooperó con él? Aquí el concepto es altruismo recíproco.

El altruismo recíproco es producto de relaciones entre sujetos sin vínculo de parentesco a partir de interacciones repetidas en el tiempo (Trivers 1971). Pero si 
éste es el caso, el concepto mismo de altruismo se vuelve problemático porque ya no tiene ese aspecto semántico que describe un acto costoso para el actor sin ganancia visible por realizarlo. Wilson (1998) añade una dificultad terminológica más al hablar de un altruismo débil, definido como aquel acto en el que el individuo ve decrecer su adaptación específica en beneficio del grupo al que pertenece, generando un bien público, entendido este último concepto como un beneficio que ayuda directamente en el proceso de adaptación a miembros de un grupo.

El altruismo humano estricto se ha observado, particularmente, con modelos matemáticos, a veces aplicados en interacciones en contextos económicos (Bowles et al. 2003; Gintis 2000, 2004), o culturales de competencia por territorio (Boyd et al. 2003). El acto altruista aquí es visto como un beneficio al grupo que produce adaptación directa al cooperador, porque aumenta las posibilidades de que ellos y el resto del grupo sobrevivan, de modo que el comportamiento altruista específico en grupos es sin autointerés. Los resultados de Boyd et al. (2003; Richerson \& Boyd 2005) muestran que, en el ejemplo de la competencia por territorio, los grupos que son más cooperadores ganan la disputa, de modo que deviene claro para al individuo de ese grupo el benficio directo en adapatación cooperando, por lo que lo hará para que en efecto su grupo sea aún más exitoso. Debido a que tales comportamientos altruistas y cooperativos son comportamientos sociales, se pueden transmitir culturalmente a través de la imitación.

¿Pero tiene el altruismo un límite? Fehr y Fischbacher (2003) dan una respuesta que, apoyada por resultados experimentales en los juegos llamados dilemas sociales, en particular en juegos de bienes públicos, es como sigue:

Strongly reciprocal individuals reward and punish in anonymous one-shot interactions. Yet they increase their rewards and punishment in repeated interactions or when their reputation is at stake. This suggests that a combination of altruistic and selfish concerns motivates them. Their altruistic motives induce them to cooperate and punishing one-shot interactions and their selfish motives induce them to increase rewards and punishment in repeated interactions or when reputation-building is possible. If this argument is correct, we should also observe that altruistic acts become less frequent as their costs increase. At a higher cost, individuals have to give up more of their own payoff to help others, so that the individuals will exhibit less altruistic behaviour for a given combination of selfish and altruistic motives. The evidence from dictator games and public good games confirms this prediction. If the own payoff that needs to be invested to produce one unit of the public good increases, subjects invest less into the public good (2003, p. 788).

Delton et al. (2011; también Clark, Cosmides \& Tooby 2010) añaden a este panorama un argumento adicional, relativo al comportamiento altruista en el contexto de toma de decisiones, en particular, en el escenario en el que el agente debe decidir si coopera o no cuando se enfrenta a la incertidumbre de si la interacción será solo de una vez con el otro agente o de repetidas ocasiones. En los modelos estándares se asume que el agente opera con un principio, que es el siguiente: si sabes que la interacción -económicaserá de solo una vez, entonces no cooperes. Sin embargo, los autores recuerdan que en la vida real, inserta en un mundo estocástico, es decir, de probabilidades, jamás un 
agente puede saber con certeza si la interacción será solo de una vez o de interacciones reiteradas, de allí que la evolución haya seleccionado en el humano, en tanto estructura de toma de decisiones, la especialización de la reciprocidad. Incluso, si el agente sabe que la interacción -económica- será de solo una ocasión, cooperará de todas maneras, porque es una conducta ya seleccionada evolutivamente. Generosidad y evitación al daño de la reputación son dos factores que afectarían esta selección. Es esta misma reputación cuidada la que la competencia argumentativa recoge, presionando para el envío de buenas razones.

\section{Conclusiones: la argumentación como proceso objetivador}

Como lo habían demostrado ejemplarmente Richerson y Boyd (2005), la cultura humana tiene un grado de sofisticación sin paralelo en el reino animal en lo que respecta a la capacidad de adaptación, siendo precisamente nuestra tendencia cooperativa superior a cualquier otra especie mamífera. Tomasello $(2008,2014)$ añade además que ninguna otra especie muestra tantos tipos de aprendizajes sociales (siendo el lenguaje y los símbolos matemáticos dos casos paradigmáticos), y con tan largos alcances y significados (siendo la construcción de instituciones sociales la evidencia más patente). La lista de logros culturales es extensa, como por ejemplo: la creación del consenso social respecto del seguimiento de normas, de entidades artificialmente valiosas (como el dinero), de los derechos y obligaciones concretas en los grupos (como el matrimonio). Lo interesante es que varias de estas tecnologías culturales y sistemas de organización se mantienen por otros fenómenos culturales de convivencia de mayor calado simbólico que, como el tabú del incesto en la organización familiar humana, son menos transparentes a la práctica consciente. Este acento de lo huamno como edificador cultural no es nuevo, en Cavalli Sforza (2004) se lee algo similar a través de su noción de animal cultural.

La diferencia clave para Tomasello se encuentra en la modificación y creación de nuevas reglas para el sostenimiento de instituciones sociales, en el marco de la intencionalidad compartida. Con este concepto, Tomasello introduce un aspecto filosófico, que integra el trabajo de Bratman (1999), Gilbert (1989), Searle (1995) y Tuomela (2007). Tomasello hace uso del término vinculándolo tripartitamente con las nociones de atención conjunta, conocimiento y motivaciones y orientación hacia fines, ideas que ya había trabajado previamente (Tomasello 1999) bajo la nomenclatura trinquete cultural. Si los agentes de una comunidad han entrenado tan repetidamente el autoconocimiento y las motivaciones orientadas hacia fines, entonces a partir de la superestructura de la intencionalidad compartida, la acumulación cultural del saber tecnológico y la creación y modificación del contrato social se hace permanente, y si ello ocurre, consecuentemente, es posible el entendimiento de la ontogénesis de la cooperación humana.

En paralelo a culturas de otras especies, la humana está orientada teleológicamente al aprovechamiento; la cooperación es su superestructura, y la transmisión del conocimiento su especificidad; la innovación, además de ser producto de la acumulación, tiene como base la imitación y la policía de normas, esto es, un especial cuidado en la vigilancia 
del cumplimiento de normas (se castiga a los egoístas o a quienes quiebran reglas de convivencia). La adaptación del pensar y actuar cooperativo del homo sapiens hace necesaria la mediación de ciertas instituciones sociales en el desarrollo de la especie que ha creado en los niños un tipo especial de inteligencia cultural.

En Why we coperate, Tomasello et al. (2009) inicia justamente su argumentación señalando que el altruismo es condición natural desde el primer año de vida. Tomasello utiliza una metáfora mercantil para referirse a los tipos de altruismo que ha encontrado en sus estudios empíricos: el humano es generoso con el bien alimentario, mientras que útil o servicial cuando le acerca a alguien un objeto fuera de su alcance, y de espíritu altruista cuando comparte información (chismes incluidos); cada uno de estos comportamientos supone diferentes costos y beneficios. Respecto de la utilidad o servicio, hay cinco razones para creer que la ayuda no es producto del aprendizaje cultural: su manifestación temprana, indiferencia ante las actitudes de estímulo y reducción de la colaboración cuando hay incentivos externos, hondas raíces evolutivas que se observan en los grandes simios, más la uniformidad en distintas culturas y su origen en las reacciones de empatía (véase también Richerson \& Henrich 2012; de Waal 2009).

Los resultados obtenidos en los estudios referentes al comportamiento de compartir información presentan, en tanto, particularidades interesantes. A medida de su inmersión cultural, el infante humano comienza a mediar normativamente en la cooperación; la propensión a compartir o ceder objetos, alimentos, etc. se bifurca bajo dos rasgos ya eminentemente sociales: (1) se comparte con mayor propensión a un miembro del propio grupo, si el beneficiario se ha mostrado amable previamente o puede devolver el favor; y (2) hay precaución en la generosidad cuando se intuye un interés oculto.

La cautela no es un rasgo exclusivo humano, pero sí el apego a las normas socialmente aceptadas respecto de la conducta amable, generosa y honesta, cuyo seguimiento se da por mor de la constitución del yo público (public self) que evita en la medida de lo posible la desaprobación y la punición sociales. De modo que la pregunta es ¿por qué los niños pasan a formar parte de la policía de normas si nadie los obliga? El misterio puede ser develado bajo la idea de intencionalidad compartida, basada en la conciencia de la interdependencia (nosotridad), en principio por identificación de los otros significativos y, luego, extendido al grupo social perteneciente.

La tolerancia parece ser entonces la causa primigenia de la evolución de la cognición humana. En este sentido tenemos dos comportamientos sensiblemente opuestos entre los primates y las crías humanas: los primeros demuestran disposición (tolerancia) a compartir alimento en tanto la presa es lo suficientemente grande para que cada participante del festín obtenga su porción (equitativa o no), o bien reparten por hostigamiento; mientras que los segundos demuestran disposición no solo a la cesión sino al reparto equitativo. El surgimiento de la cultura en la evolución de los humanos la encontramos en la emergencia del mutualismo que, a su vez, tiene como fundamento la tolerancia en el contexto de la satisfacción grupal de alimento.

¿Por qué debiera ser vista la competencia argumentativa como resultado natural de este devenir evolutivo? Porque, como se ha dicho, es una tecnología que beneficia a 
todos los interlocutores (el que da buenas razones y, con ello, representaciones adecuadas, asegura mayor reputación; el que las recibe puede optar por una nueva representación que le hace desechar la equivocada); porque la repetición del acto de dar buenas razones genera expectativas positivas que circularmente mantienen la competencia; porque se objetiva un proceso al que se puede recurrir por aquellos que no detentan una influencia de otra índole en los grupos; porque es una tecnología colectiva inteligente que renueva constantemente la tolerancia básica que hace fluir nuestros mecanismos comunicativos. Y porque, una vez satisfecha la necesidad física de alimento, permite modelar el conflicto que genera satisfacer la necesidad simbólica.

\section{Referencias bibliográficas}

Axelrod, Robert (1997), The Complexity of Cooperation. Princeton, NJ: Princeton University Press.

Axelrod, R., \& W. Hamilton (1981), “The evolution of cooperation”, Science 211: 1390-1396.

Barth, E. \& E. Krabbe (1982), From axiom to dialogue. A philosophical study of logics and argumentation. Berlin: de Gruyter.

Bermúdez, José (2003), Thinking without Words. New York: Oxford University Press.

Boehm, Christopher (2012), Moral Origins. The Evolution of Virtue, Altruism, and Shame. New York: Basic Books.

Bowles, S. \& H. Gintis (2011), A Cooperative Species. Human Reciprocity and its Evolution. Princeton: Princeton University Press.

(2004), "The Evolution of Strong Reciprocity: Cooperation in Heterogeneous Populations", Theoretical Population Biology 65: 17-28.

Boyd, R., H. Gintis, S. Bowles \& P. Richerson (2003), "The evolution of altruistic punishment". Proc. Natl. Acad. Sci. 100: 3531-3535.

Bratman, Michael (1999), Faces of Intentionality. Cambridge: Cambridge University Press.

(2014), Shared Agency. A planning Theory of Acting Together. New York: Oxford University Press.

Carruthers, Peter (2006), The Architecture of the Mind. Oxford: Oxford University Press.

(2011), The Opacity of Mind. An Integrative Theory of Self-Knowledge. New York: Oxford University Press.

Cavalli Sforza, Luigi (2004), L'evoluzione della cultura. Turin: Codice edizioni.

Clark, H., Cosmides, L. \& J. Tooby (2010), "Coevolution of cooperation, causal cognition and mindreading", Communicative \& Integrative Biology 3, 6: 522524.

Corbalis, Michael (2011), The Recursive Mind. The Origins of Human Language, Though, and Civilization. Princeton: Princeton University Press. 
Dahanukar, N. \& M. Watve (2009), "Group Selection and Reciprocity among Kin", The Open Biology Journal 2: 66-79.

Dawkins, Richard (1976), The selfish gene. New York: Oxford University Press.

Delton, A., M. Krasnow, L. Cosmides, \& J. Tooby (2011), "Evolution of direct reciprocity under uncertainty can explain human generosity in one-shot encounters", PNAS 108 (32): 13335-13340.

Dugatkin, Lee (2006), The Altruism equation. Seven Scientists search for the origins of goodness. Boston: Princeton University Press.

Eemeren, F. van \& R. Grootendorst (2004), A Systematic Theory of Argumentation. The Pragma-dialectical Approach. New York: Cambridge University Press.

Evans, Jonathan (2010), Thinking Twice. Two Minds in One Brain. New York: Oxford University Press.

Fehr, E. \& Fischbacher, U. (2003), “The nature of human altruism”, Nature 425: 785791.

Frank, Robert (1988), Passions within reason. The strategic role of the emotions. New York: W.W. Norton.

Freeman, James (2005), Acceptable premises. An epistemic approach to an Informal Logic Problem. Cambridge: Cambridge University Press.

Hamilton, William (1996), Narrow Roads of Gene Land vol. 1: Evolution of Social Behaviour. Oxford: Oxford University Press.

Henrich, N. \& J. Henrich (2007), Why Humans Cooperate. A cultural and evolutionary explanation. New York: Oxford University Press.

Hwanga, S. \& S. Bowles (2012), "Is altruism bad for cooperation?", Journal of Economic Behavior \& Organization 83: 330-341.

Gilbert, Margaret (1989), On Social Facts. London: Routledge.

Gilbert, Michael (2007), "Natural Normativity: Argumentation Theory as Engaged Discipline", Informal Logic 27 (2): 149-161.

Gintis, Herbert (2000), "Strong Reciprocity and Human Sociality", J. Theor. Biol 206: 169-179.

(2004), "The evolutionary roots of human hyper-cognition", J. Bioecon. DOI 10.1007/s10818-012-9140-6.

Godfrey-Smith, Peter (2009), Darwinian Populations and Natural Selection. New York: Oxford University Press.

Godfrey-Smith, P. \& K. Yegnashankaran (2011), "Reasoning as deliberative in function but dialogic in structure and origin", Behavioral and Brain Sciences 34(2): 80.

Griebel, U., \& D. Oller (2008), "Evolutionary Force Favoring Communicative Flexibility", en D. Oller \& U. Griebel, eds., Evolution of Communicative Flexibility. Cambridge, Mass.: The MIT Press, pp. 9-40.

Johnson, Ralph (2000), Manifest Rationality. A Pragmatic Theory of Argument. Mahwah: Lawrence Erlbaum Associates. 
Kahneman, Daniel (2011), Thinking Fast and Slow. London: Allen Lane.

Krebs, J. \& N. Davies (1993), An Introduction to Behavioural Ecology. Oxford: Blackwell.

Kock, Christian (2007), "The domain of rhetorical argumentation”, en F. van Eemeren, J. Blair, C. Willard \& B. Garssen, eds., Proceedings of the Sixth Conference of the International Society for the Study of Argumentation. Amsterdam: Sic Sat; pp. $785-788$.

Lewis, David (1969), Convention. Cambridge, MA: Harvard University Press.

Maynard-Smith, J. \& D. Harper (2003), Animal Signals. New York: Oxford University Press.

Mercier, H. \& D. Sperber (2011), "Why do human reason? Arguments for an argumentative theory", Behavioral and Brain Sciences 34(2): 57-74.

Mercier, H., M. Deguchi, J-V. van der Henst \& H. Yama (2015), "The benefits of argumentation are cross-culturally robust: The case of Japan", Thinking \& Reasoning, http://dx.doi.org/10.1080/13546783.2014.1002534

Naess, Arne (1953), Interpretation and Preciseness. A contribution to the theory of communication. Oslo: Skrifter utgitt ar der norske videnskaps academie.

Perelman, Ch. \& L. Olbrechts-Tyteca (1958), The New Rhetoric. A Treatise on Argumentation. Notre Dame: University of Notre Dame Press.

Ratnieks, Francis (2006), "The evolution of cooperation and altruism: the basic conditions are simple and well known", J. Evol. Biol. 19: 1413-1414.

Richerson, P. \& R. Boyd (2005), Not by Genes alone. How Culture Transformed Human Evolution. Chicago: Chicago University Press.

Richerson, P., R. Boyd \& J. Henrich (2003), “The cultural evolution and cooperation", en P. Hammerstein, ed., Genetic and Cultural Evolution of Cooperation. Cambridge, Mass.: MIT Press; pp. 357-388.

Richerson, P. \& J. Henrich (2012), "Tribal Social Instincts and the Cultural Evolution of Institutions to Solve Collective Action Problems", Cliodynamics 3: 38-80.

Rigotti, Edo. (2009), "Whether and how classical topics can be revived within contemporary argumentation theory", en F. van Eemeren \& B. Garssen, eds., Pondering on problems of argumentation. Dordrecht: Springer, pp. 157-178.

Santibáñez, Cristián (2012), “Mercier and Sperber's Argumentative Theory of Reasoning. From the Psychology of Reasoning to Argumentation Studies", Informal Logic 32 (1): 132-159.

(2015), "Robustez como categoría para el análisis de la cognición: el caso de la competencia argumentativa", Cinta de Moebio 52: 60-68.

Searle, John (1995), The Construction of Social Reality. New York: The Free Press.

(2010), Making the Social World. The Structure of Human Civilization. New York: Oxford University Press.

Skyrms, Brian (2004), The Stag Hunt and the Evolution of Social Structure. New York: Cambridge University Press. 
(2014), Social Dynamics. New York: Oxford University Press.

Sterelny, Kim (2003), Thought in a hostile world: The evolution of human cognition. Malden, MA: Blackwell Publishing.

(2012), The Evolved Apprentice. How Evolution Made Humans Unique. Cambridge, Mass: The MIT Press.

Tindale, Christopher (2004), Rhetorical Argumentation. New York: Sage.

Tomasello, Michael (1999), The Cultural Origins of Human Cognition. Cambridge, MA: Harvard University Press.

(2008), Origins of Human Communication. Cambridge, Mass.: The MIT Press.

(2014), A Natural History of Human Thinking. Cambridge, Mass.: Harvard University Press.

Tomasello, M. et al. (2005), "Understanding and sharing intentions: The origins of cultural cognition”, Behavioral \& Brain Sciences 28: 675-735.

(2009), Why We Cooperate. Cambridge, Mass.: The MIT Press.

Tomasello, M., A. Melis, C. Tennie, E. Wyman \& E. Herrmann (2012), “Two Key Steps in the Evolution of Human Cooperation: The Interdependence Hypothesis", Current Anthropology 53 (6): 673-692

Toulmin, Stephen (1958), The Uses of Argument. Cambridge: Cambridge University Press. 114.

(2004), "Reasoning in Theory and Practice", Informal Logic 24, 2: 111-

Toulmin, S., R. Rieke \& A. Janik (1979), An Introduction to Reasoning. New York: Macmillan.

Trivers, Robert (1971), “The evolution of Reciprocal Altruism”, Quarterly Review of Biology 46: 35-57.

Tuomela, Raimo (2007), The Philosophy of Sociality. The Shared point of View. New York: Oxford University Press.

Waal, Frans de (2009), The Age of Empathy. Nature's Lessons for a Kinder Society. London: Souvenir Press.

Walton, Douglas (2013), Methods of Argumentation. New York: Cambridge University Press.

West, S., A. Griffin \& A. Gardner (2007), "Evolutionary Explanations for Cooperation”, Current Biology 17: 661-672.

(2006), "Social semantics: altruism, cooperation, mutualism, strong reciprocity and group selection", European Society for Evolutionary Biology 20: 415-432.

Wilson, Eduard (1998), Consilience. The Unity of Knowledge. New York: Alfred A. Kopf.

Wynne-Edwards, Vero (1962), Animal Dispersion in Relation to Social Behaviour. Edinburgh: Oliver \& Boyd. 
Zimmerman, M., R. Mcelreath \& P. Richerson (2003), "The Evolution of Cooperation", en A. Hastings \& L. Gross, eds., Encyclopaedia of Theoretical Ecology. Berkeley: University of California Press; pp.155-162. 